\title{
Histological observation on the initial stage of vascular invasion into the sec- ondary ossification of murine femoral epiphyseal cartilage
}

Keiji Hashimoto ${ }^{1,2}$, Tomoka Hasegawa ${ }^{1}$, Tomomaya Yamamoto $^{1,7}$, Hiromi Hongo ${ }^{1}$, Yimin ${ }^{9}$, Miki Abe ${ }^{1}$, Alireza Nasoori ${ }^{1}$, Ko Nakanishi ${ }^{3,4}$, Haruhi Maruoka ${ }^{1,4}$, Yasuhito Morimoto ${ }^{1,5}$, Keisuke Kubota ${ }^{1,6}$, Tomohiro Shimizu $^{8}$, Mai Haraguchi ${ }^{1}$, Masahiko TaKahata ${ }^{8}$, Norimasa Iwasaki ${ }^{8}$, Minqi Li $^{10}$, Toshiaki Fujisawa ${ }^{2}$, and Norio AMIZUKA ${ }^{1}$

${ }^{1}$ Developmental Biology of Hard Tissue, ${ }^{2}$ Dental Anesthesiology, ${ }^{3}$ Biomaterials and Bioengineering, ${ }^{4}$ Orthodontics, ${ }^{5}$ Periodontology and Endodontology, ${ }^{6}$ Oral Functional Prosthodontics, Graduate School of Dental Medicine and Faculty of Dental Medicine, Hokkaido University, Sapporo, Japan; ${ }^{7}$ Northern Army Medical Unit, Camp Makomanai, Japan Ground Self-Defense Forces, Sapporo, Japan; ${ }^{8}$ Department of Orthopaedic Surgery, ${ }^{9}$ Central Research Institute, Faculty of Medicine and Graduate School of Medicine, Hokkaido University, Sapporo, Japan; and ${ }^{10}$ Shandong Provincial Key Laboratory of Oral Biomedicine, The School of Stomatology, Shandong University, Jinan, China

(Received 4 April 2021; and accepted 31 May 2021)

\begin{abstract}
It remains unknown whether the histology of vascular invasion during secondary ossification of epiphyseal cartilage is the same as that seen in primary ossification; we examined the initial processes of vascular invasion of secondary ossification in the murine femora. Many endomucin-immunoreactive blood vessels gathered at the central region of the articular surface, and buds of soft tissue, including glomerular loops of endomucin-immunoreactive blood vessels and TNALPaseimmunopositive osteoblastic cells accompanied by TRAP-positive osteoclasts, had begun to invade the epiphyseal cartilage. The invading soft tissues formed cartilage canals displaying MMP9 immunoreactivity in the tip region, and cartilaginous collagen fibrils were not visible in the vicinity of the vascular wall of the blood vessels. Thus, the histological profile marked by invading glomerular vasculature and the erosion of the cartilage matrix near the vascular walls during secondary ossification differs from that seen during primary ossification.
\end{abstract}

\section{INTRODUCTION}

Long bone develops through a cartilage model, which provides the primary and secondary centers of ossification. There is a lag time between the occurrence of primary and secondary ossification in the cartilage model. In mice, primary ossification centers are formed at the middle of cartilage anlage in the

Address correspondence to: Tomoka Hasegawa DDS, $\mathrm{PhD}$.

Developmental Biology of Hard Tissue, Graduate School of Dental Medicine and Faculty of Dental Medicine, Hokkaido University, Kita-13, Nishi-7, Kita-Ku, Sapporo, Japan

Tel: +81-11-706-4226, Fax: +81-11-706-4226

E-mail: hasegawa@den.hokudai.ac.jp fetal stage. After birth, secondary ossification occurs in the extremities of the long bones, forming epiphyseal cartilage and articular cartilage. The histological process of them is quite similar; both histological processes are achieved by vascular invasion into cartilage accompanied by osteoblastic migration. However, there are some substantial differences between them. When the cartilage anlage shows a mineralized hypertrophic zone, i.e., primary ossification center, the mineralized bone collar comes to surround the midshaft of the cartilage anlage (Sasaki et al. 2000), which is followed by appearance of osteoclasts and osteoblastic cells, as well as blood vessels outside close to the cortical areas of midshaft of bone. After the vascular invasion into the primary ossification center, endochondral bone formation begins with the replacement of epiphyseal cartilage 
into bone. In the hypertrophic zone of the epiphyseal cartilage, transverse partitions of cartilaginous columns are incompletely mineralized, therefore easily permitting the vascular invasion accompanied by migration of osteoblastic cells, osteoclasts, and other cell types (Amizuka et al. 2012; Tsuchiya et al. 2020). In contrast, the longitudinal intercolumnar septa are well-mineralized and serve as scaffolds of primary trabeculae spicules which are distributed uniformly in areas parallel to the longitudinal axis. Consequently, mineralized intercolumnar septa are exposed, and then, migrating osteoblasts will localize on the mineralized cartilage to form new bone via matrix vesicle-mediated mineralization and abundant collagen synthesis (Marks and Odgren 2002; Ozawa et al. 2008; Amizuka et al. 2012; Hasegawa et al. 2016; Hasegawa 2018). Therefore, the longitudinal distribution of the mineralized cartilage matrix appears to be essential for the formation of the primary trabecular bones.

In contrast, ossification of the secondary center is not preceded by covering of mineralized bone collar (Floyd et al. 1987). Previous reports observed the canals running through the unmineralized epiphyseal cartilage, which serve as a path for invading blood vessels and osteogenic cells (Levene 1964; DelgadoBaeza et al. 1992). After cartilaginous canals reach the deeper portion, the secondary ossification begins and extends radially from the middle of the epiphysis. However, the cellular interplay and mechanisms that trigger the formation of vascular canals in specific regions of the perichondrium are still veiled.

Recent studies have suggested the existence of a blood vessel subtype specific to bone during endochondral ossification, which highly expresses both CD31 and endomucin, maintains perivascular osteoprogenitors, and couples angiogenesis to osteogenesis (Kusumbe et al. 2014; Ramasamy et al. 2014). More recently, we have consistently demonstrated metaphyseal blood vessels positive for not only endomucin but also EphB4, indicating that endomucin-positive vascular endothelial cells in metaphyses are of venous sinusoid origin (Tsuchiya et al. 2020). At the erosion zone between epiphyseal cartilage and subchondral bone, vascular endothelial cells extended long cytoplasmic processes, with finger-like fine processes at the invasion tip inserting cartilage matrix. We and others have also demonstrated that MMP9 would be involved in vascular invasion in the histological process of endochondral bone formation (Vu et al. 1998; Engsig et al. 2000; Kojima et al. 2013). Therefore, it seems likely that vascular endothelial cells may extend their cytoplasmic pro- cesses with MMP9, when invading into the cartilage. However, the precious histochemical approach has not verified yet whether endomucin-positive vascular endothelial cells would pierce their cytoplasmic processes into the articular surface of the epiphyseal cartilage to form the cartilage canals prior to secondary ossification. In addition, there are only a few reports on the accompanying cell types following invading vascular endothelial cells during the initial stage of the secondary ossification.

Taken together, in this study, we have chronologically examined the histology of the initial steps of endomucin-positive vascular endothelial cells and accompanying cell types that invade into the epiphyseal cartilage.

\section{MATERIALS AND METHODS}

Animals and tissue preparation. Postnatal mice at 1, $3,5,7,10,12$ and 14 days after birth (CLEA Japan Co. Ltd, Tokyo) were used in this study ( $n=6$ for each). The experimental protocol was approved by the Hokkaido University Animal Care and Use Committee which is accredited by the Association for Assessment and Accreditation of Laboratory Animal Care (approval No. 20-0023).

All mice were euthanized with an intraperitoneal injection of a combination of anesthetic agents prepared with $0.3 \mathrm{mg} / \mathrm{kg}$ of medetomidine, $4.0 \mathrm{mg} / \mathrm{kg}$ of midazolam, and $5.0 \mathrm{mg} / \mathrm{kg}$ of butorphanol. Femora were extracted from the mice, then immersed in $4 \%$ paraformaldehyde solution diluted in a $0.067 \mathrm{M}$ phosphate buffer ( $\mathrm{pH} 7.4$ ) for $24 \mathrm{~h}$ at $4^{\circ} \mathrm{C}$. After fixation, left femora were decalcified with $10 \%$ ethylenediamine tetraacetic disodium salt (EDTA-2Na, $\mathrm{pH}$ 7.4) solution between 3 days and 3 weeks before paraffin-embedding. Five-micrometer-thick paraffin sections parallel to the sagittal line of the femora were made, and all the paraffin sections subjected to the following immunohistochemistry were photographed under a Nikon Eclipse E800 microscope (Nikon Instruments Inc. Tokyo, Japan). Light microscopic images were acquired with a digital camera (Nikon DXM1200C, Nikon). Right femora without decalcification were post-fixed with $1 \%$ osmium tetroxide with a $0.1 \mathrm{M}$ cacodylate buffer for $4 \mathrm{~h}$ at $4^{\circ} \mathrm{C}$, dehydrated in ascending acetone solutions, and embedded in epoxy resin (Epon 812; TAAB, Berkshire, UK) (Hasegawa et al. 2017; Hasegawa 2018). The semi-thin sections were subjected to toluidine blue staining. Ultrathin sections were prepared with an ultramicrotome, and then stained with uranyl acetate and lead citrate or left unstained for transmission 
electron microscopy (TEM) examination (Hitachi H-7100; Hitachi Co. Ltd, Tokyo, Japan) at $80 \mathrm{kV}$.

Immunohistochemistry for tissue-nonspecific alkaline phosphatase (TNALPase), endomucin, cathepsin K, and matrix metallo-proteinase (MMP) 9. Dewaxed paraffin sections were immersed in phosphate buffered saline (PBS) containing $0.3 \% \mathrm{H}_{2} \mathrm{O}_{2}$ for $30 \mathrm{~min}$ to block endogenous peroxidase. To reduce non-specific binding, $1 \%$ bovine serum albumin (BSA; Seologicals Proteins Inc. Kankakee, IL) in PBS (1\% BSA-PBS) was applied to the sections for $20 \mathrm{~min}$. The sections treated above were incubated with rabbit polyclonal antisera against TNALPase (Oda et al. 1999; Hasegawa et al. 2019a) diluted at 1:300 with $1 \%$ BSA-PBS for $2 \mathrm{~h}$ at room temperature (RT), and were then incubated with horseradish peroxidase (HRP)-conjugated goat anti-rabbit IgG (DakoCytomation, Glostrup, Denmark) at a dilution of $1: 100$ for $1 \mathrm{~h}$ at RT. For endomucin detection, the serial sections were reacted with rat antibody against endomucin (Santa Cruz Biotechnology, Inc., Dallas, TX) at a dilution of $1: 100$ overnight at $4^{\circ} \mathrm{C}$, and were then incubated with HRP-conjugated anti-rat IgG (Zymed Laboratories Inc., South San Francisco, $\mathrm{CA}$ ) at a dilution of $1: 100$. For cathepsin $\mathrm{K}$ immunohistochemistry, the sections were incubated with mouse anti-human cathepsin K (Daiichi Fine Chemical Co., Ltd., Takaoka, Japan) at a dilution of $1: 200$ overnight, and then incubated with HRP-conjugated anti-mouse IgG (Chemicon International Inc., Temecula, CA) at a dilution of $1: 100$. As with MMP9 immunodetection, the histologically-serial sections were reacted with goat antibody against mouse MMP9 (R\&D Systems, Inc., Mineapolis, MN) at a dilution of $1: 100$, and then with HRP-conjugated anti-goat IgG (American Qualex Antibodies, San Clemente, CA; 1:100). Immune complexes were visualized using 3, 3'-diaminobenzidine tetrahydrochloride (Dojindo Laboratories, Kumamoto, Japan).

Enzyme histochemistry for tartrate-resistant acid phosphatase (TRAP). Tartrate-resistant acid phosphatase (TRAP) was detected as previously described (Hasegawa et al. 2019b); in short, slides were rinsed with PBS and incubated in a mixture of $2.5 \mathrm{mg}$ of naphthol AS-BI phosphate (Sigma-Aldrich Co. LLC, St. Louis, MS), $18 \mathrm{mg}$ of red violet LB salt (Sigma), and $100 \mathrm{mM} \mathrm{L}(+)$ tartaric acid $(0.76 \mathrm{~g}$; Nacalai Tesque, Inc., Kyoto, Japan) diluted in $30 \mathrm{~mL}$ of a $0.1 \mathrm{M}$ sodium acetate buffer ( $\mathrm{pH} 5.0$ ) for $15 \mathrm{~min}$ at $37^{\circ} \mathrm{C}$. Following immunostaining, TRAP detection was conducted as described above.

\section{RESULTS}

Chronological change of histology of soft tissue invasion into the ossification center in the femoral epiphyseal cartilage

Between days 1-3 after birth, the sagittal sections of the distal epiphyseal cartilage appeared slightly concave on the articular surface, accompanied by relatively thickened perichondrium (Fig. 1A, B, E, F). At day 5, a small bud of soft tissue focally invaginated into the articular surface (Fig. 1C, G). At around day 7 , the invading bud of soft tissue inserted towards the center of the epiphyseal cartilage, forming a cartilage canal without spreading transversely parallel to the articular surface (Fig. 1D, H). Chondrocytes close to the invading soft tissue did not show hypertrophic profiles until day 7 after birth. After day 10, the invading soft tissues appeared to extend in all directions, forming bony trabeculae when they reached the center of the epiphyseal cartilage (Fig. 1I-K, L-N). Chondrocytes adjacent to the invading soft tissues at this stage were hypertrophied (Fig. 1L). On the sagittal sections, during days $1-14$, the invasion of the soft tissues took place at the central region (not from multiple sites) of the articular surface of the epiphyseal cartilage.

Chronological change in the distribution of endomucin-positive blood vessels and MMP9-reactive soft tissues invading into the epiphyseal cartilage

At days 1-3 after birth, endomucin-positive blood vessels tended to accumulate on the central region of the articular surface (Fig. 2A, B). At around days $5-7$, the glomerular loops of endomucin-positive blood vessels were observed in the small buds of soft tissues invading into the epiphyseal cartilage (Fig. 2C, D). Between days 10 and 14, many endomucin-positive blood vessels were seen in the invading soft tissues (Fig. 2E-G).

At days 1-3, weak immunoreactivity of MMP9 could be seen on the articular surface (Fig. 3A, B), while at around days 5-7, intense MMP9-immunoreactivity was observed mainly in the tips of the invading soft tissues (Fig. 3C, D). From days 10-14, MMP9-immunoreactivity was seen at the periphery, rather than inside of the soft tissues (Fig. 3E-G).

Immunolocalization of endomucin, TNALPase, MMP9, TRAP, and cathepsin K, and TEM observation at days 1 and 5 of vascular invasion into the epiphyseal cartilage

Next, we observed, at high resolution, the distribution of endomucin-positive blood vessels, TNALPase- 

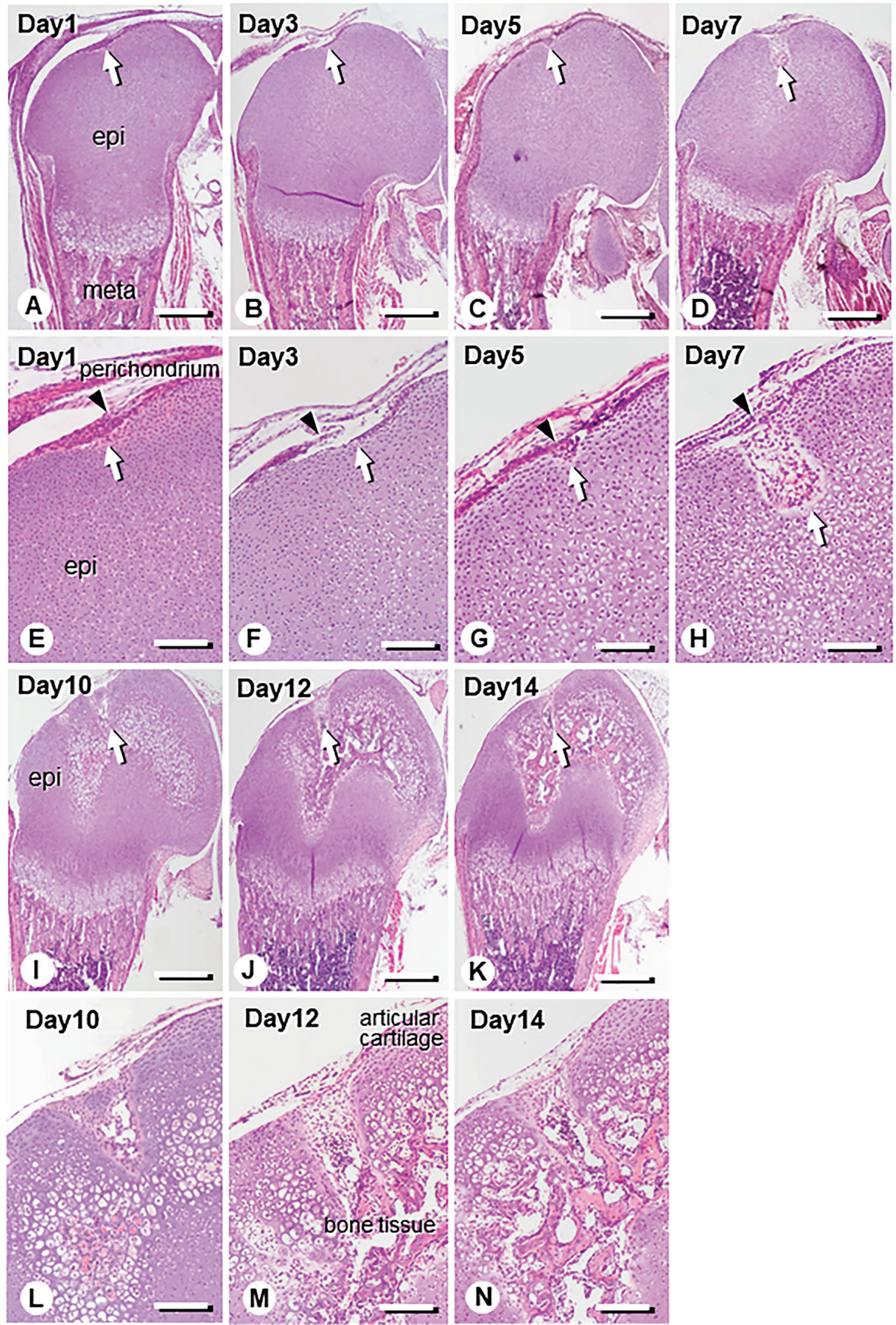
reactive osteoblastic cells, TRAP-positive osteoclasts, and MMP9-immunoreactivity. At day 1, the central region of the articular surface was slightly concave, showing many endomucin-positive blood vessels located on the articular surfaces (Fig. 4A, B). When observed under a higher resolution of epoxy resin-embedded specimens and ultrathin sections under TEM, capillaries were located parallel to the articular surface, accompanied by fibroblastic articular cells (Fig. 6A, B). TEM observation demonstrated that blood vessels did not appear to extend their cytoplasmic processes vertically into the cartilage matrix (Fig. 6C). The capillaries showed smooth surface of the walls, and there were fine collagen fibrils close to the capillaries and facing chondrocytes (Fig. 6D). TNALPase-reactive osteoblastic cells focally accumulated in the central region of the articular surface, and there were a few numbers of TRAP-positive osteoclasts in the corresponding area of TNALPase-reactive cells (Fig. 4C, D). Weak MMP9-immunoreactivity was also observed in cells located on the central region of the articular cartilage (Fig. 4E, F).

At day 5, a small bud of soft tissue could be seen, which initiated invasion of the cartilage (Fig. 5A). Endomucin-positive blood vessels accumulated to form glomerular loops in the invading buds (Fig. 5B). There was broad but weak TNALPase-reactivity in both perichondrium and chondrocytes (Fig. 5C). The invading buds of the soft tissue included a few TRAP-positive osteoclasts (Fig. 5D) which, however, did not show cathepsin K-immunoreactivity (Fig. 5E). The invading buds of soft tissue revealed MMP9-immunoreactivity in the invading region, but not the whole region of the soft tissue (Fig. 5F). When observed at a higher resolution, most blood vessels of the buds showed the vascular structure facing the cartilage matrix (Fig. 6E, F). TEM observation demonstrated fine collagen fibrils close to the capillary walls of vascular endothelial cells, which showed smooth walls without extending cytoplasmic processes into the cartilage (Fig. 6G). The meshwork of cartilaginous collagen fibrils seemed to dis- appear in the vicinity of the vascular wall.

Immunolocalization of endomucin, TNALPase, MMP9, TRAP, and cathepsin K at days 7 and 14 of vascular invasion into the epiphyseal cartilage

At day 7, the invading buds of soft tissue showed glomerular loops of endomucin-positive blood vessels inside (Fig. 7A, B). MMP9-immunoreactivity was seen mainly in the peripheral region of the invading soft tissue; MMP9 appeared to be immunolocalized not only in the region of endomucinpositive blood vessels, but also in other cells located in the tip areas of the soft tissue (Fig. 7C). Although there were several TRAP-positive osteoclasts among TNALPase-positive cells, cathepsin K-immunoreactive osteoclasts were hardly detected in the invading soft tissues (Fig. 7D-F).

At day 14, the region of the invading tip, featuring mixed spicules of cartilage and bone, contained many endomucin-positive blood vessels (Fig. 7G, H). MMP9-immunoreactivtity was seen in the periphery of the invading soft tissue, especially in the blood vessels and osteoclasts (Fig. 7I). There were many TRAP-positive/cathepsin K-negative osteoclasts among TNALPase-positive osteoblastic cells (Fig. 7J-L).

\section{DISCUSSION}

Our study aimed to evaluate the chronological histology of the initial processes of vascular invasion during secondary ossification of the murine epiphyses, and consequently demonstrated the following:

1) In the sagittal section of the distal epiphyseal cartilage of mice, the singular vascular invasion at the central region of the articular surface was observed at postnatal days $1-14$.

2) Endomucin-immunopositive blood vessels accumulated on the central region of the articular surface at day 1 and then formed a glomerular loop in the invading buds of soft tissue.

3) TNALPase-immunopositive osteoblastic cells

Fig. 1 Chronological change of histology of soft tissue invasion into the femoral epiphyseal cartilage. Panels A-D, and I$\mathbf{K}$ are low magnified images of femoral epiphyses at days $1,3,5,7,10,12$, and 14 after birth. Panels $\mathbf{E}-\mathbf{H}$ and $\mathbf{L}-\mathbf{N}$ are highly magnified images of $\mathbf{A}-\mathbf{D}$ and $\mathbf{I}-\mathbf{K}$, respectively. White arrows in panels $\mathbf{A}-\mathbf{K}$ indicate the tip of the invading soft tissue. At days 1 and 3, the articular surface of the epiphyseal cartilage is slightly concave (white arrows), accompanied by relatively thickened perichondrium (arrowheads) (E, F). At days 5 and 7, a small bud of soft tissue (arrowheads) focally invaginates into the articular surface, and then inserts into the deeper region of the epiphyseal cartilage that does not contain obviously hypertrophied chondrocytes (white arrows, G, H). At day 10, the invading soft tissues reach deeply in the epiphyseal cartilage, and the surrounding chondrocytes hypertrophy (L). At days 12 and 14, apparent bony trabeculae have formed in the epiphyseal cartilage (M, N). meta: metaphysis, epi: epiphysis. Bars, A-D, I-K: $250 \mu \mathrm{m}, \mathrm{E}-\mathrm{H}, \mathrm{L}-\mathrm{N}$ : $100 \mu \mathrm{m}$ 

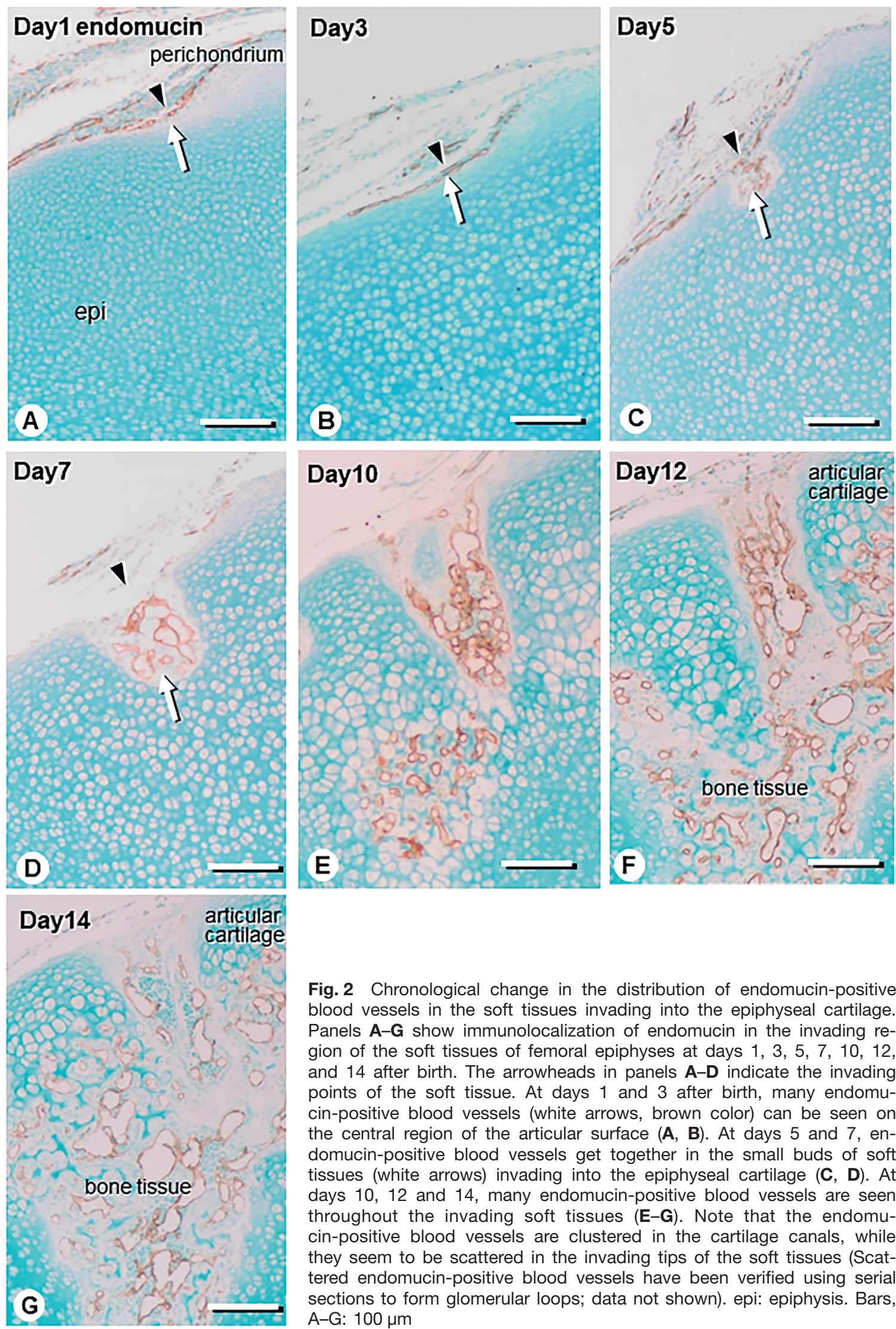

Fig. 2 Chronological change in the distribution of endomucin-positive blood vessels in the soft tissues invading into the epiphyseal cartilage. Panels A-G show immunolocalization of endomucin in the invading region of the soft tissues of femoral epiphyses at days 1, 3, 5, 7, 10, 12, and 14 after birth. The arrowheads in panels A-D indicate the invading points of the soft tissue. At days 1 and 3 after birth, many endomucin-positive blood vessels (white arrows, brown color) can be seen on the central region of the articular surface (A, B). At days 5 and 7 , endomucin-positive blood vessels get together in the small buds of soft tissues (white arrows) invading into the epiphyseal cartilage (C, D). At days 10, 12 and 14, many endomucin-positive blood vessels are seen throughout the invading soft tissues $(\mathbf{E}-\mathbf{G})$. Note that the endomucin-positive blood vessels are clustered in the cartilage canals, while they seem to be scattered in the invading tips of the soft tissues (Scattered endomucin-positive blood vessels have been verified using serial sections to form glomerular loops; data not shown). epi: epiphysis. Bars, A-G: $100 \mu \mathrm{m}$ 

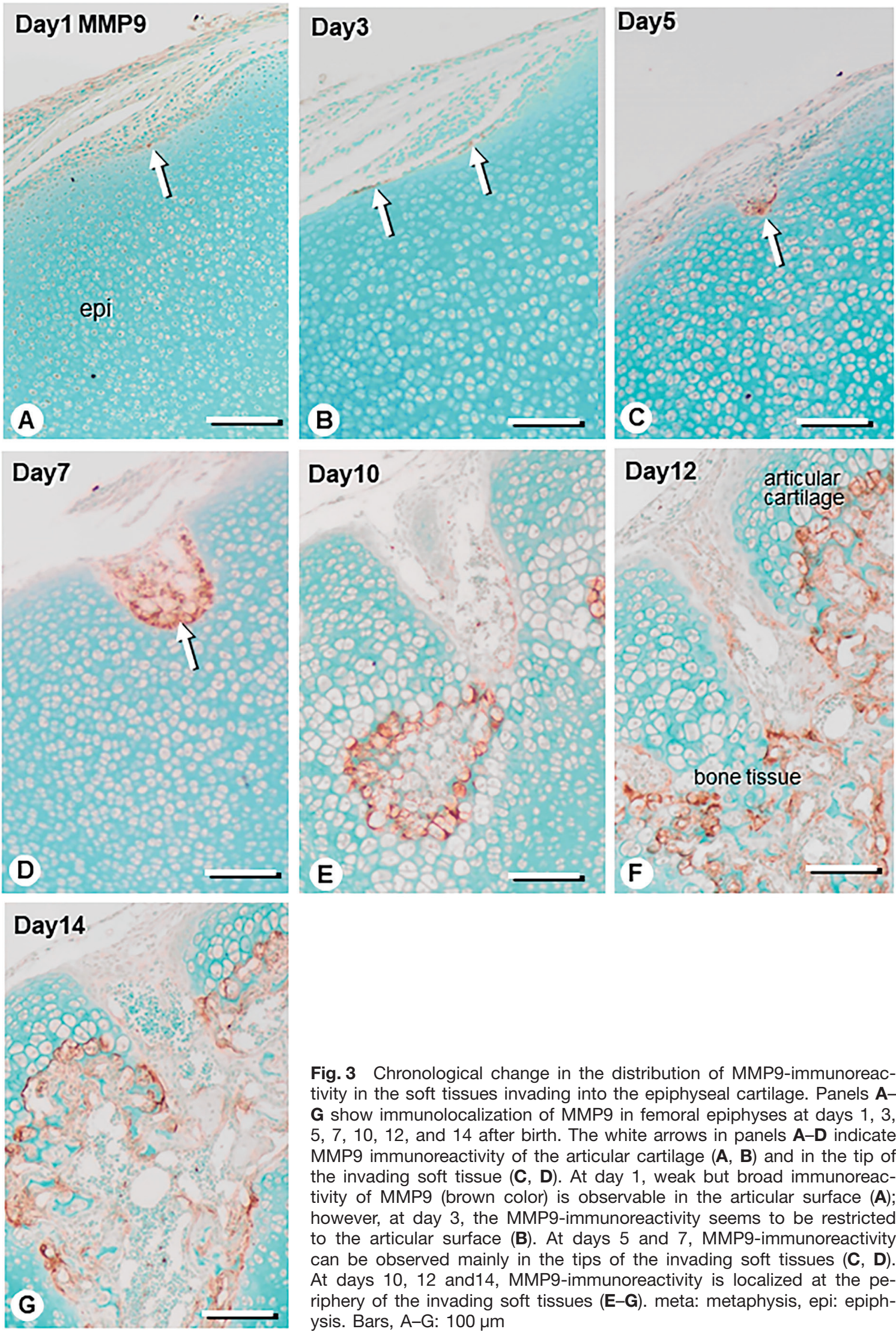

Fig. 3 Chronological change in the distribution of MMP9-immunoreactivity in the soft tissues invading into the epiphyseal cartilage. Panels AG show immunolocalization of MMP9 in femoral epiphyses at days 1, 3, $5,7,10,12$, and 14 after birth. The white arrows in panels A-D indicate MMP9 immunoreactivity of the articular cartilage (A, B) and in the tip of the invading soft tissue (C, D). At day 1, weak but broad immunoreactivity of MMP9 (brown color) is observable in the articular surface (A); however, at day 3, the MMP9-immunoreactivity seems to be restricted to the articular surface (B). At days 5 and 7, MMP9-immunoreactivity can be observed mainly in the tips of the invading soft tissues (C, D). At days 10, 12 and14, MMP9-immunoreactivity is localized at the periphery of the invading soft tissues $(\mathbf{E}-\mathbf{G})$. meta: metaphysis, epi: epiphysis. Bars, A-G: $100 \mu \mathrm{m}$ 

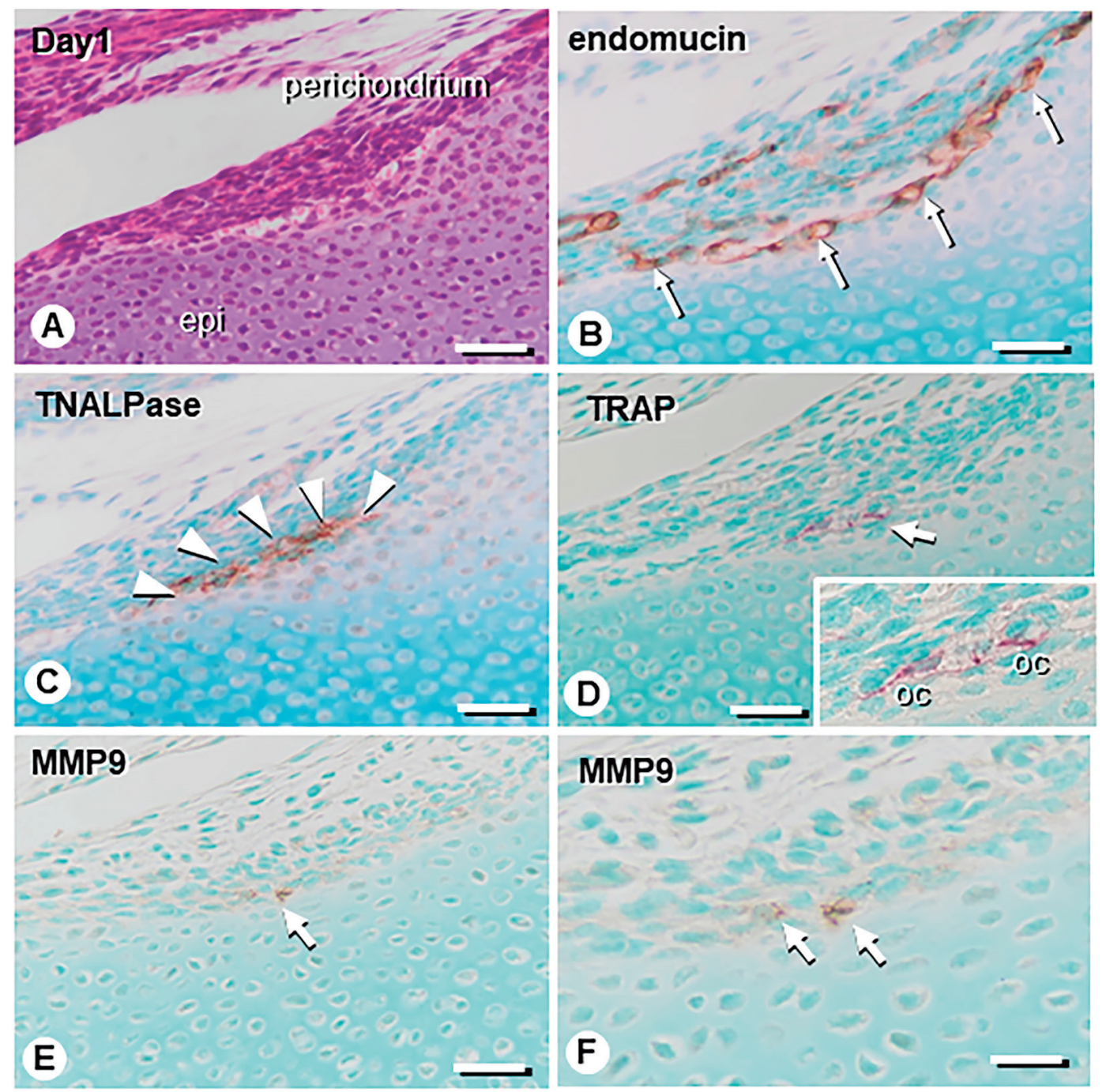

Fig. 4 Immunolocalization of endomucin, TNALPase, TRAP and MMP9 of the soft tissue invading into the epiphyseal cartilage at day 1. At day 1 , the central region of the articular surface is slightly concave, being covered with thickened perichondrium (A). There are many endomucin-immunopositive blood vessels (white arrows) located on the articular surfaces (B). Focal accumulation of TNALPase-reactive cells (white arrowheads, brown color, C) and TRAP-positive osteoclasts (an arrow in D) can be seen on the articular surface of the epiphysis. An inset of panel $\mathbf{D}$ shows higher magnification TRAP-positive osteoclasts (oc). Weak MMP9-immunoreactivity (arrow, brown color) can be seen on the articular surface (E). Highly magnified images show MMP9-immunopositivity (arrows) in cells located on the articular surface (F). epi: epiphysis. Bars, A-E: $30 \mu \mathrm{m}, \mathrm{F}: 10 \mu \mathrm{m}$

focally accumulated in the region that endomucinpositive blood vessels gathered at day 1, and TRAPpositive osteoclasts appeared in the corresponding region.

4) After day 5, the invading soft tissue showed MMP9-immunoreactivity in not only endomucinpositive blood vessels but also other cell types in the tip areas.

Taken together, the buds of soft tissue, as a lump of many cell types, rather than vascular endothelial cells solely, appear to invade into the epiphyseal cartilage to establish cartilage canals. Therefore, the histological process of vascular invasion of secondary ossification and primary ossification, i.e., endochondral bone formation at the boundary between epiphyseal cartilage and metaphyseal bone, seems similar, but there are several differences in the cellular events between them, as shown in this study.

We and others have reported that vascular endothelial cells of the endochondral bone formation at the boundary between epiphyseal cartilage and me- 

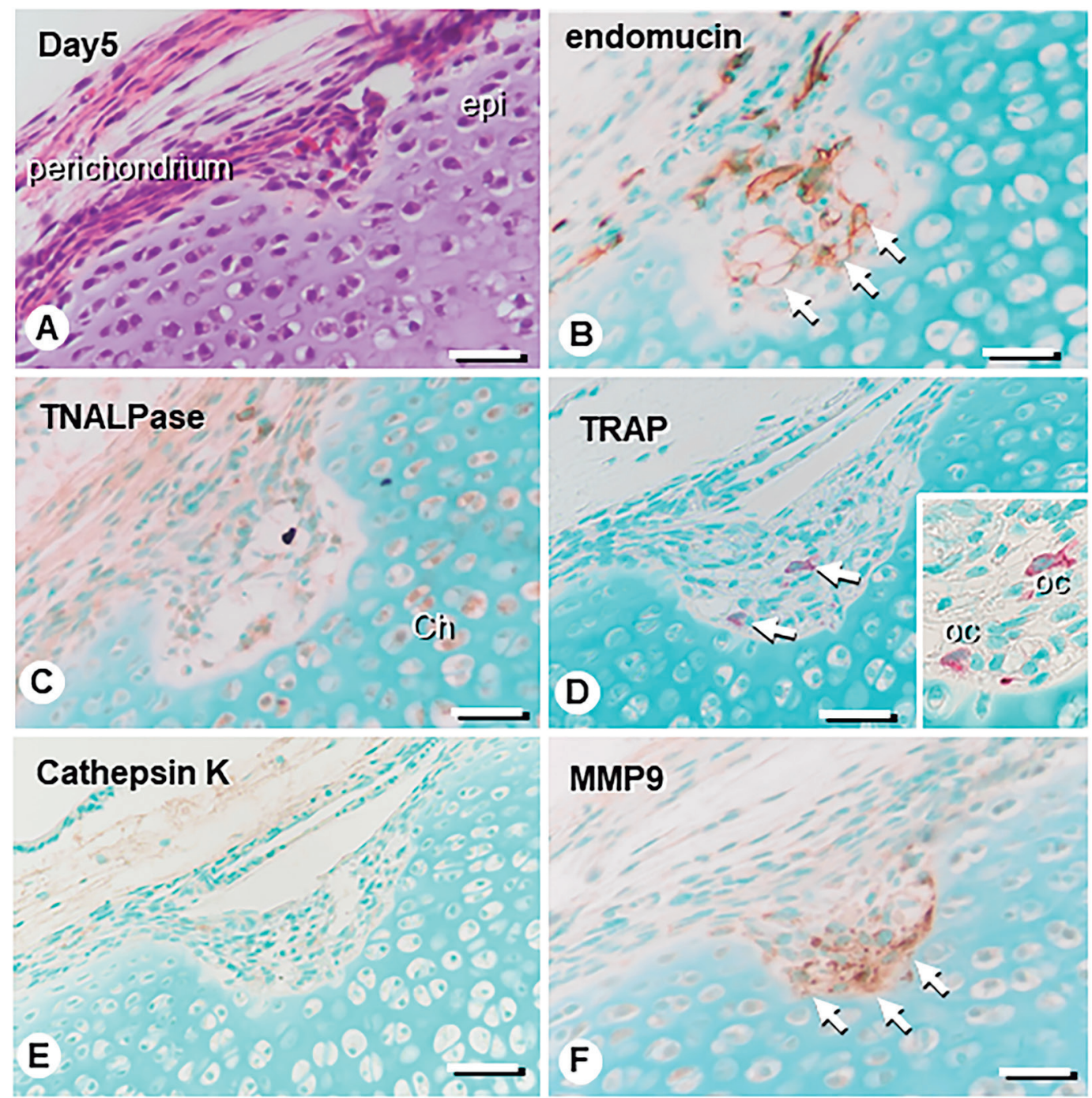

Fig. 5 Immunolocalization of endomucin, TNALPase, TRAP, cathepsin K and MMP9 of the soft tissue invading into the epiphyseal cartilage at day 5. At day 5 , a small bud of soft tissue has been formed, inserting into the epiphyseal cartilage (A). Endomucin-positive blood vessels (brown color) gather in the invading buds (white arrows in B). Broad but weak TNALPase-reactivity (faint brown color) can be seen in both perichondrium and chondrocytes (Ch) (C). A few TRAP-positive osteoclasts (white arrows, red color) are observable in the invading soft tissue (D). An inset of panel $\mathbf{D}$ is a highly magnified image of TRAP-positive osteoclasts (oc). However, there cannot be seen cathepsin K-immunoreactive osteoclasts (E). MMP9-immunoreactivity (brown color, white arrows) is intense in the tip of the invading region but not throughout the entire region of the soft tissue $(\mathbf{F})$. Note that panels $\mathbf{A}-\mathbf{F}$ are derived from the same specimen at day 5 , but only panels $\mathbf{D}$ and $\mathbf{E}$ show serial sections. epi: epiphysis. Bars, A-F: $30 \mu \mathrm{m}$

taphyseal bone extend fine cytoplasmic processes to pierce the transverse partitions of the cartilage columns (Amizuka et al. 2012; Tsuchiya et al. 2020). In addition, MMP9-immunoreactivity was shown to be localized to the invading blood vessels and surrounding osteoclasts, which resorb mineralized cartilaginous matrix (Kojima et al. 2013). Under TEM observation in this study, the vascular endothelial cells of the invading soft tissue did not have cytoplasmic processes that extend into the facing carti- laginous matrix (Fig. 6G). However, even without piercing by the cytoplasmic processes, the cartilaginous collagen fibrils seemed to disappear, indicating digestion of the cartilage matrix in the vicinity of the vascular walls and cell bodies of the vascular endothelial cells. Consistently, previous literature has proposed the loop structure as a modular structure of invading blood vessels into the epiphyseal cartilage (Ganey et al. 1992). Taken together, it seems likely that vascular endothelial cells would 

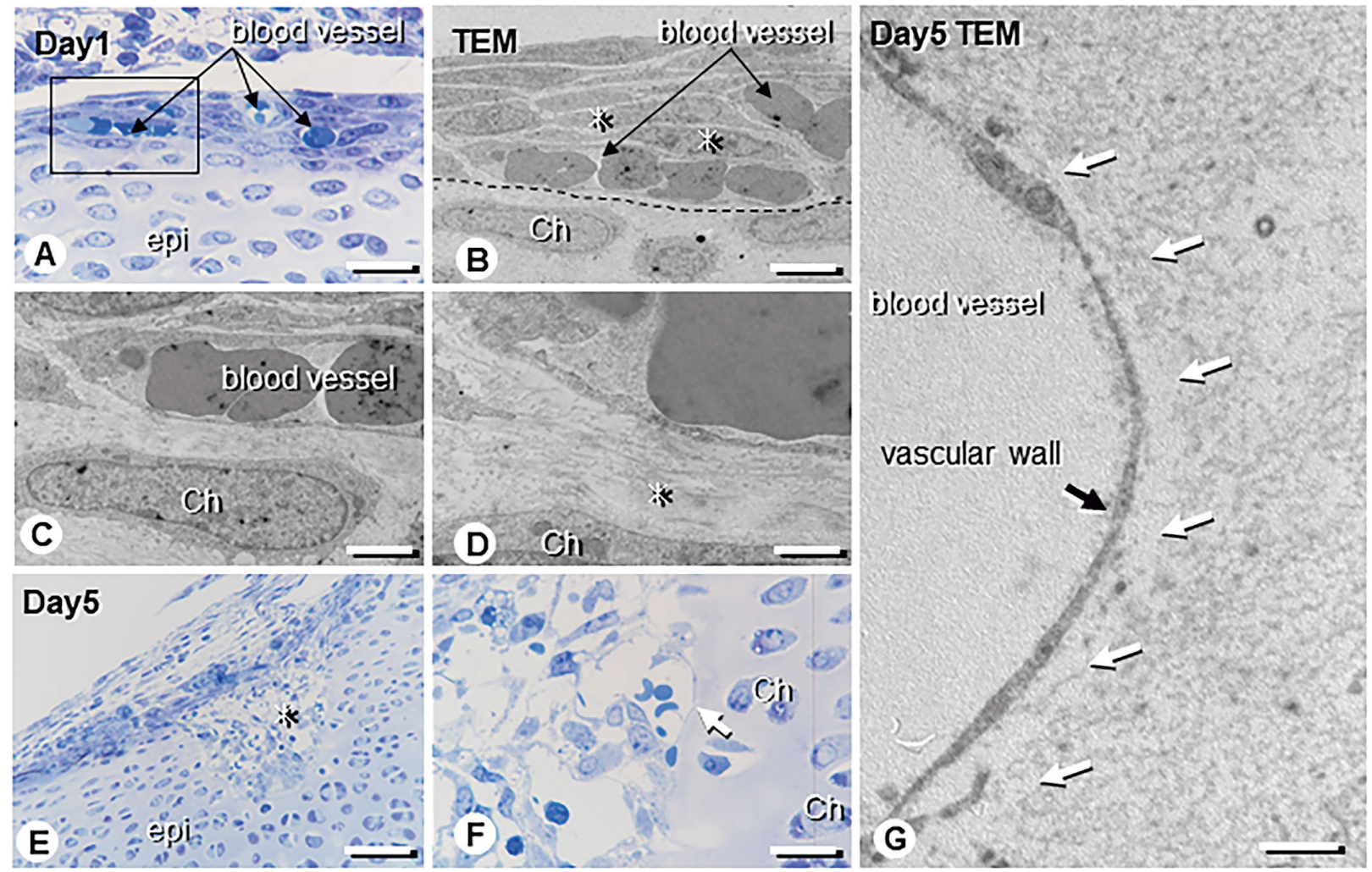

Fig. 6 Light microscopic images with high resolution and TEM observations of the invading soft tissue at days 1 and 5 . At day 1, blood vessels can be seen on the articular surface, without invading into the epiphyseal cartilage (A). Under TEM observation, blood vessels are located on the articular surface, being accompanied by overlying fibroblastic articular cells (asterisks, B). Note the dotted lines indicative of the articular surface. TEM observation at higher magnification demonstrates that blood vessels do not extend the cytoplasmic processes into the cartilage matrix (C), and also that the wall of the blood vessels, which is seen as capillaries without perivascular cells, is smooth. Note the fine collagen fibrils (asterisk) between the capillary and facing chondrocytes (D). At day 5, the bud of soft tissue (asterisk in E) invaginates into the cartilage including blood vessels (E, F). Panel $\mathbf{F}$ is a highly magnified image of $\mathbf{E}$. The blood vessels in the buds demonstrate the vascular structure facing the cartilage matrix (an arrow, F). TEM observation demonstrates fine collagen fibrils close to the capillary walls of vascular endothelial cells $(\mathbf{G})$. However, the meshwork of cartilaginous collagen fibrils is not visible in the vicinity of the vascular wall (white arrows, G). Epi: epiphysis, Ch: chondrocyte. Bars, A, F: $10 \mu \mathrm{m}, \mathrm{B}: 4 \mu \mathrm{m}, \mathrm{C}: 2 \mu \mathrm{m}, \mathrm{D}$, G: $1 \mu \mathrm{m}, \mathrm{E}: 30 \mu \mathrm{m}$

digest and erode cartilaginous matrix by piercing with their cytoplasmic processes for endochondral bone formation of primary ossification, while by the vascular walls of blood vessels in the process of secondary ossification.

In endochondral bone formation of primary ossification, the transverse partitions of the hypertrophic zone are incompletely mineralized, consequently permitting vascular invasion, while the longitudinal intercolumnar septa are well-mineralized and serve as scaffolds of primary trabeculae spicules (Amizuka et al. 2012; Kojima et al. 2013; Tsuchiya et al. 2020). Thus, chondrocyte differentiation and accompanying mineralization are well-organized in the processes of endochondral bone formation of primary ossification. In the initial step of secondary ossifi- cation, in our observation, chondrocytes close to the invading soft tissues did not hypertrophy until day 7 in the femoral epiphyseal cartilage of mice, still featuring the histological profile of resting chondrocytes: spherical, compact cell shape and abundant accumulation of glycogens (See Fig. 6). After the invading soft tissues reached the central region of the epiphyseal cartilage at day 10 , the chondrocytes adjacent to the invading tissues started to become hypertrophied for formation of the trabecula bones. Therefore, cellular states of chondrocytes and the histological properties of cartilage matrices that would be subjected to vascular invasion seem to differ between primary ossification and secondary ossification.

As shown in Fig. 5, TRAP-positive osteoclasts 

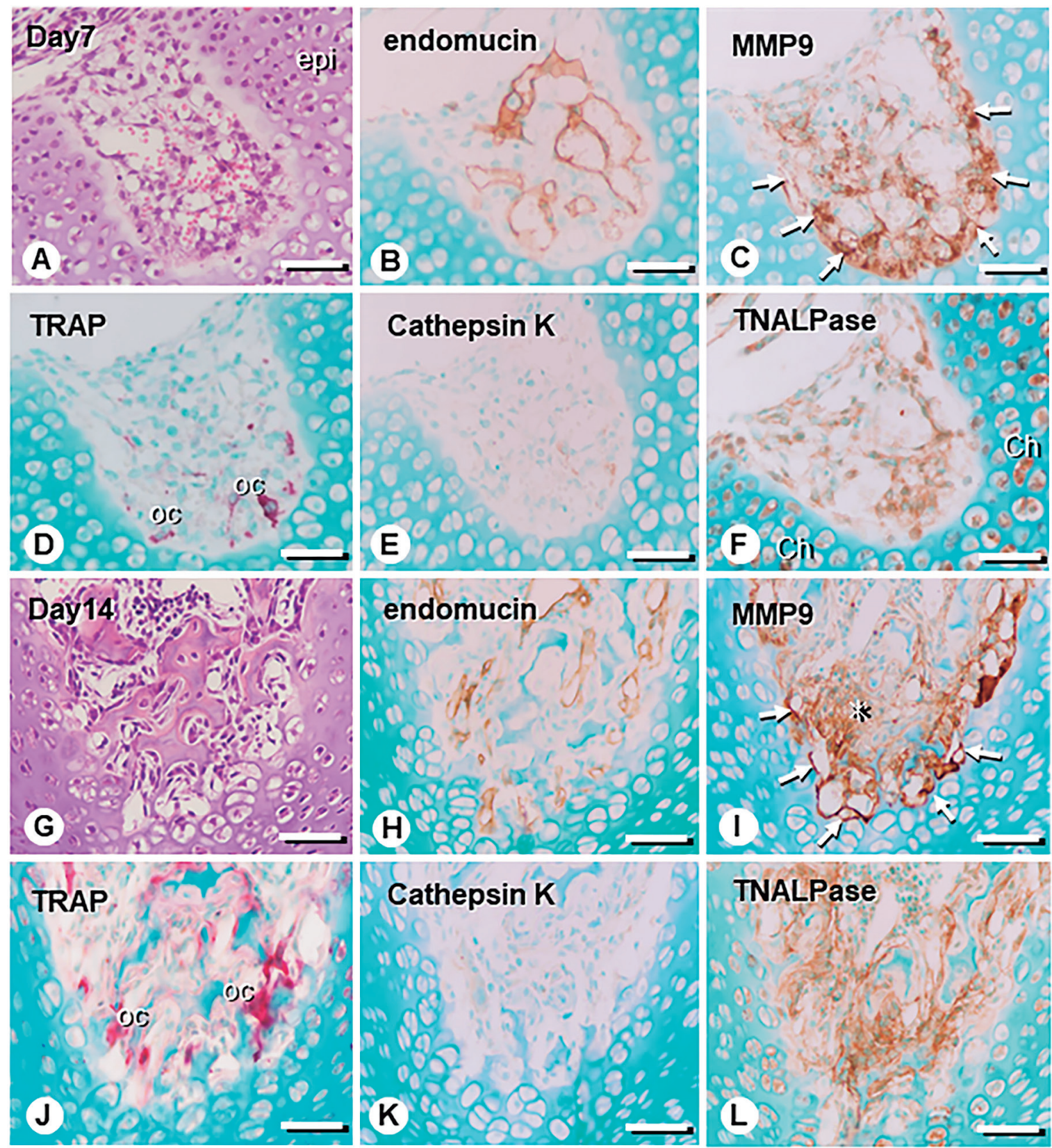

Fig. 7 Immunolocalization of endomucin, MMP9, TRAP, cathepsin K and TNALPase of the soft tissues invading into the epiphyseal cartilage at days 7 and 14. At day 7, an invading bud of soft tissue shows the gathering of endomucin-positive blood vessels (brown color) (A, B). MMP9-immunoreactivity can be seen in the peripheral region of and inside the invading soft tissue (white arrows, C). Although several TRAP-positive osteoclasts (red color, oc) can be observed (D), but, cathepsin $\mathrm{K}$-immunoreactive osteoclasts are hardly detected in the invading soft tissues (E). Faint TNALPase-immunopositivity (weak brown color) are seen in the invading soft tissue and surrounding chondrocytes (Ch) (F). At day 14 , the region of the invading tip features mixed spicules of cartilage and bone (G). Many endomucin-positive blood vessels (brown color) are seen throughout the soft tissue $(\mathbf{H})$. MMP9-immunoreactivtity (intense brown color) is observed not only in the periphery of the invading soft tissue, but also fibrillar structures (an asterisk) inside the soft tissue (I). Many TRAP-positive osteoclasts (red color, J) are seen, but they do not show cathepsin K-immunopositivity (K). Many TNALPase-positive osteoblastic cells (brown color) are present throughout the soft tissue (L). epi: epiphysis. Bars, A-L: $30 \mu \mathrm{m}$ 
did not show any immunoreactivity for cathepsin $\mathrm{K}$, which is specific for digesting type I collagen (Inaoka et al. 1995; Kojima et al. 2013). Consistently, we have previously observed that osteoclasts at the boundary of epiphyseal cartilage display an intense immunoreactivity of MMP9 rather than cathepsin K, while those in the trabecular termini exhibit intense cathepsin K (Kojima et al. 2013). This implies that osteoclasts synthesize proteolytic enzymes according to the chemical properties of the surrounding matrix. Likely, MMP9 was seen not only in blood vessels but also other cell types in the invading tip of the soft tissue as seen in our study and others (Davoli et al. 2001), though MMP9 was shown to be localized mainly in invading vascular endothelial cells and accompanying osteoclasts during endochondral bone formation (Kojima et al. 2013). Therefore, cells in the invading soft tissues may have the potential to synthesize proteolytic enzymes including MMP9, appropriate to digesting surrounding cartilage matrix. However, it is reasonable that other proteolytic enzymes would be involved in digestion of cartilaginous matrix. Davoli et al. (2001) have described that collagenase 3 was restricted to the center of the canal, whereas MMP9 extended to the nearby portion of the canal walls. Alvarez et al. (2005) have demonstrated the localization of genes encoding of MMPs 13 and 14, also in the invading soft tissues. Therefore, there must be interplays of proteolysis among many cell types in the invading soft tissue.

It is still veiled why and how endomucin-positive blood vessels accumulate in the sagittal center of articular surface at days 1-3 in our murine model. Several reports have demonstrated the immunolocalization of vascular endothelial growth factor (VEGF) in chondrocytes of the epiphyseal cartilage close to soft tissue insertion (Morini et al. 2004; Allerstorfer et al. 2010). Therefore, angiogenic factors such as VEGF may induce the migration of endomucinpositive blood vessels onto the articular surfaces. In addition, the region that accumulated endomucinpositive blood vessels also revealed TNALPase-activity. This may suggest the osteogenic differentiation of mesenchymal cells on and over the articular cartilage. We conjecture that these TNALPase-reactive cells may be incorporated in the buds of the soft tissues, participating in future secondary ossification in the epiphyses. Osteoclasts may be theoretically unnecessary for cartilage invasion of the soft tissue buds, because no mineralized region of epiphyseal cartilage at day 5 was detected by von Kossa staining (data not shown). Taken together, osteoblastic cells and osteoclasts that would be accompanied by invading blood vessels into the epiphyses appear to be differentiated from the beginning of the soft tissue invasion, rather than just before forming mineralized bone matrix deep inside of the epiphyses.

\section{Acknowledgments}

This study was partially supported by the Grants-in Aid for Scientific Research of Japan Society for the Promotion of Science (Hasegawa T).

\section{CONFLICT OF INTEREST}

No potential conflicts of interest exist.

\section{REFERENCES}

Allerstorfer D, Longato S, Schwarzer C, Fischer-Colbrie R, Hayman AR, et al. (2010) VEGF and its role in the early development of the long bone epiphysis. $J$ Anat 216, 611-624.

Alvarez J, Costales L, Serra R, Balbín M and López JM (2005) Expression patterns of matrix metalloproteinases and vascular endothelial growth factor during epiphyseal ossification. $J$ Bone Miner Res 20, 1011-1021.

Amizuka N, Hasegawa T, Oda K, Luiz de Freitas PH, Hoshi K, et al. (2012) Histology of epiphyseal cartilage calcification and endochondral ossification. Front Biosci 4, 2085-2100.

Davoli MA, Lamplugh L, Beauchemin A, Chan K, Mordier S, et al. (2001) Enzymes active in the areas undergoing cartilage resorption during the development of the secondary ossification center in the tibiae of rats aged 0-21 days: II. Two proteinases, gelatinase B and collagenase-3, are implicated in the lysis of collagen fibrils. Dev Dyn 222, 71-88.

Delgado-Baeza E, Nieto-Chaguaceda A, Miralles-Flores C and Santos-Alvarez I (1992) Cartilage canal growth: Experimental approach in the rat tibia. Acta Anat 145, 143-148.

Engsig MT, Chen QJ, Vu TH, Pedersen AC, Therkidsen B, et al. (2000) Matrix metalloproteinase 9 and vascular endothelial growth factor are essential for osteoclast recruitment into developing long bones. J Cell Biol 151, 879-889.

Floyd WE III, Zaleske DJ, Schiller AL, Trahan C and Mankin HJ (1987) Vascular events associated with the appearance of the secondary center of ossification in the murine distal femoral epiphysis. J Bone Joint Surg Am 69, 185-190.

Ganey TM, Love SM and Ogden JA (1992) Development of vascularization in chondroepiphysis of the rabbit. J Orthopedic Res 10, 496-510.

Hasegawa T, Yamamoto T, Tsuchiya E, Hongo H, Tsuboi K, et al. (2016) Ultrastructural and biochemical aspects of matrix vesicle-mediated mineralization. Jpn Dent Sci Rev 52, 6374.

Hasegawa T, Endo T, Tsuchiya E, Kudo A, Zhao S, et al. (2017) Biological application of focus ion beam-scanning electron microscopy (FIB-SEM) to the imaging of cartilaginous fibrils and osteoblastic cytoplasmic processes. J Oral Biosci 59, 55-62.

Hasegawa T (2018) Ultrastructure and biological function of matrix vesicles in bone mineralization. Histochem Cell Biol 149, 289-304.

Hasegawa T, Miyamoto Y, Abe M, Qiu Z, Yamamoto T, et al. 
(2019a) Histochemical examination on principal collagen fibers in periodontal ligaments of ascorbic acid-deficient ODS-od/od rats. Microscopy 68, 349-358.

Hasegawa T, Yamamoto T, Sakai S, Miyamoto Y, Hongo H, et al. (2019b) Histological effects of the combined administration of eldecalcitol and a parathyroid hormone in the metaphyseal trabeculae of ovariectomized rats. J Histochem Cytochem 67, 169-184.

Inaoka $\mathrm{T}$, Bilbe $\mathrm{G}$, Ishibashi $\mathrm{O}$, Tezuka $\mathrm{K}$, Kumegawa $\mathrm{M}$, et al. (1995) Molecular cloning of human cDNA for cathepsin K: novel cysteine proteinase predominantly expressed in bone. Biochem Biophys Res Commun 206, 89-96.

Kojima T, Hasegawa T, de Freitas PH, Yamamoto T, Sasaki M, et al. (2013) Histochemical aspects of the vascular invasion at the erosion zone of the epiphyseal cartilage in MMP-9-deficient mice. Biomed Res (Tokyo) 34, 119-128.

Kusumbe AP, Ramasamy SK and Adams RH (2014) Coupling of angiogenesis and osteogenesis by a specific vessel subtype in bone. Nature 507, 323-328.

Levene C (1964) The pattern of cartilage canals. J Anat 98, 515538.

Marks SC Jr and Odgren PR (2002) The structure and development of the skeleton. In: Principles of Bone Biology (ed by Bilezikian JP, Raisz LG, Rodan GA). pp3-15, Academic press, New York.

Morini S, Continenza MA, Ricciardi G, Gaudio E and Pannarale
L (2004) Development of the microcirculation of the secondary ossification center in rat humeral head. Anat Rec A Discov Mol Cell Evol Biol 278, 419-427.

Oda K, Amaya Y, Fukushi-Irie M, Kinameri Y, Ohsuye K, et al. (1999) A general method for rapid purification of soluble versions of glycosylphosphatidylinositol-anchored proteins expressed in insect cells: An application for human tissue-nonspecific alkaline phosphatase. J Biochem 126, 694699.

Ozawa H, Hoshi K and Amizuka N (2008) Current concepts of bone mineralization. J Oral Biosci 50, 1-14.

Ramasamy SK, Kusumbe AP, Wang L and Adams RH (2014) Endothelial Notch activity promotes angiogenesis and osteogenesis in bone. Nature 507, 376-380.

Sasaki T, Amizuka N, Irie K, Ejiri S and Ozawa H (2000) Localization of alkaline phosphatase and osteopontin during matrix mineralization in the developing cartilage of coccygeal vertebrae. Arch Histol Cytol 63, 271-824.

Tsuchiya E, Hasegawa T, Hongo H, Yamamoto T, Abe M, et al. (2020) Histochemical assessment on the cellular interplay of vascular endothelial cells and septoclasts during endochondral ossification in mice. Microscopy (Oxf). 70, 201-214.

$\mathrm{Vu}$ T, Shipley HJM, Bergers G, Berger JE, Helms JA, et al. (1998) MMP-9/gelatinase B is a key regulator of growth plate angiogenesis and apoptosis of hypertrophic chondrocytes. Cell 93, 411-422. 